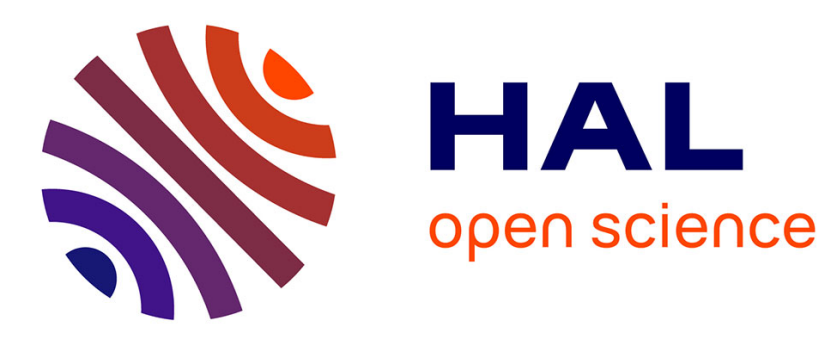

\title{
Stability of non-conservative elastic structures under additional kinematics constraints
}

Noël Challamel, François Nicot, Jean Lerbet, Félix Darve

\section{To cite this version:}

Noël Challamel, François Nicot, Jean Lerbet, Félix Darve. Stability of non-conservative elastic structures under additional kinematics constraints. Engineering Structures, 2010, 32 (10), pp.3086-3092. 10.1016/j.engstruct.2010.05.027 . hal-00833665

\section{HAL Id: hal-00833665 https://hal.science/hal-00833665}

Submitted on 26 Dec 2019

HAL is a multi-disciplinary open access archive for the deposit and dissemination of scientific research documents, whether they are published or not. The documents may come from teaching and research institutions in France or abroad, or from public or private research centers.
L'archive ouverte pluridisciplinaire HAL, est destinée au dépôt et à la diffusion de documents scientifiques de niveau recherche, publiés ou non, émanant des établissements d'enseignement et de recherche français ou étrangers, des laboratoires publics ou privés. 


\title{
Stability of non-conservative elastic structures under additional kinematics constraints
}

\author{
Noël Challamel ${ }^{\mathrm{a}, *}$, François Nicot ${ }^{\mathrm{b}}$, Jean Lerbet ${ }^{\mathrm{c}}$, Félix Darve ${ }^{\mathrm{d}}$ \\ a INSA de Rennes, Université Européenne de Bretagne, Laboratoire de Génie Civil et Génie Mécanique, 20, avenue des Buttes de Coësmes, 35043 Rennes cedex, France \\ ${ }^{\mathrm{b}}$ CEMAGREF, Unité de Recherche Erosion Torrentielle - Neige et Avalanches, 2, rue de la papeterie, 38042 St. Martin d'Heres cedex, France \\ ${ }^{\mathrm{c}}$ Université d'Evry Val d'Essonne, UFR Sciences et Technologie, 40, rue du Pelvoux CE 1455, 91020 Evry Courcouronnes Cedex, France \\ ' INPG, Laboratoire “Sols, Solides, Structures-Risques", BP 53, 38041 Grenoble cedex 9, France
}

In this paper, the specific effect of additional constraints on the stability of undamped non-conservative elastic systems is studied. The stability of constrained elastic system is compared to the stability of the unconstrained system, through the incorporation of Lagrange multipliers. It is theoretically shown that the second-order work criterion, dealing with the symmetric part of the stiffness matrix corresponds to an optimization criterion with respect to the kinematics constraints. More specifically, the vanishing of the second-order work criterion corresponds to the critical kinematics constraint, which can be interpreted as an instability direction when the material stability analysis is considered (typically in the field of soil mechanics). The approach is illustrated for a two-degrees-of-freedom generalised Ziegler's column subjected to different constraints. We show that a particular kinematics constraint can stabilize or destabilize a non-conservative system. However, for all kinematics constraints, there necessarily exists a constraint which destabilizes the non-conservative system. The constraint associated to the lowest critical load is associated with the second-order criterion. Excluding flutter instabilities, the second-order work criterion is not only a lower bound of the stability boundary of the free system, but also the boundary of the stability domain, for all mixed perturbations based on proportional kinematics conditions.

\section{Stability of constrained linear systems}

Stability of elastic structures is a branch of engineering that has been well developed since the 1960s, especially in the presence of non-conservative loading [1,2]. Most of the published studies in this area have focused on circulatory loadings (see recently the flutter phenomenon in arch instabilities [3]). In this paper, we theoretically investigate the influence of additional kinematics constraints on the stability of non-conservative elastic systems. The additional constraints could be suggested for instance to stabilize the system in structural mechanics. Specifically, we are interested by the optimal buckling load with respect to some constraints on the kinematics parameters (minimization and maximization). A lot of work has been devoted to the optimization of elastic structures (see for instance [4,5] or more recently [6]). However, it seems that the non-conservative stability problem under general kinematics constraints still merits some

\footnotetext{
* Corresponding author. Tel.: +33022323 84 78; fax: +33 0223238491 .

E-mail addresses: noel.challamel@insa-rennes.fr (N. Challamel), francois.nicot@grenoble.cemagref.fr (F. Nicot), jean.lerbet@ibisc.univ-evry.fr (J. Lerbet), felix.darve@inpg.fr (F. Darve).
}

complementary investigations. Such kinematics constraints could be also typically met in soil mechanics, where isochoric conditions for instance are usual. We show that the second-order work criterion, dealing with the symmetric part of the stiffness matrix, is strongly related to the stability boundary of the constrained system. All instabilities considered in this paper are divergence instabilities. Extension of such a procedure to stability theory in the general case (including flutter instabilities-loss of stability in which the structure is oscillating at the critical load) is a more difficult task.

Equations of motion of the free undamped linear system can be written as:

$\underline{\underline{M}} \underline{\ddot{x}}+\underline{K} \underline{x}=\underline{0}$

where the matrix $\underline{K}$ is generally a non-symmetric matrix (in the case of non-conservative elastic systems). The matrix $\underline{K}(p)$ generally depends on a loading factor denoted by $p$. Matrix $\overline{\bar{M}}$ is assumed to be a positive definite matrix. $x$ is the perturbation of dimension $n$. For the undamped system, the stability criteria are greatly simplified. In a conservative system, the matrix $K$ is symmetric. Stability (in the sense of Lyapunov) of the equilibrium can be investigated by means of the Lagrange-Dirichlet criterion. 
The positive definitiveness of the stiffness matrix $K$ is easily checked from Sylvester's criterion [7]. The loss of positive definiteness is reached when the determinant of one of the submatrices of Sylvester's criterion vanishes. In the general case (conservative or non-conservative systems), the stability domain can be checked from application of Routh-Hurwitz criteria (see for instance [3]). In case of divergence instabilities, the boundary between stability and instability is generally given by the singularity condition (see for instance [4] or [8]):

$\operatorname{det}(\underline{\underline{K}})=0$.

For conservative systems, the stability criterion is given by the vanishing of the determinant of the stiffness matrix. Considering the stiffness matrix $\underline{K}$ or its symmetric part $\underline{K}^{S}$ is equivalent for conservative systems $\left(\operatorname{det}(\underline{\underline{K}})=\operatorname{det}\left(\underline{\underline{K}}^{S}\right)\right)$. For non-conservative elastic systems, the static criterion $(\operatorname{det}(\underline{\underline{K}})=0)$ also holds in cases of divergence instabilities. For this type of instability, the second-order work criterion $\left(\operatorname{det}\left(\underline{K}^{S}\right)=0\right)$ constitutes a lower bound of the stability boundary of the undamped system [9].

$\operatorname{det}\left(\underline{\underline{K}}^{S}\right)=0$

where $\underline{K}^{S}$ is the symmetric part of $\underline{K}$. This criterion is sometimes called the second-order work criterion, with reference to the second-order differential energy [10]:

$\forall \underline{\delta x}, \quad \underline{\delta x^{T}} \underline{\underline{K}} \underline{\delta x}=\underline{\delta x^{T}} \underline{\underline{K}}^{S} \underline{\delta x} \geq 0 \Rightarrow \operatorname{det}\left(\underline{\underline{K}}^{S}\right) \geq 0$.

The boundary of such a criterion leads to the determinant of the vanishing of the symmetric part of the matrix $\underline{K}$ according to Eq. (3).

In cases of flutter instability, no theorem guarantees the lower bound status of the second-order work criterion [11]. In other words, only flutter instabilities can potentially precede the secondorder work criterion.

We would like to investigate the properties of such a dynamical system in the presence of an additional kinematics constraint, given by the holonomic constraint:

$\underline{\alpha}^{T} \cdot \underline{x}=0$.

This constraint does not affect the trivial equilibrium position $\underline{x}=\underline{0}$. The reader can be reported to the recent work of Roithmayr and Hodges [12] for the rigorous introduction of nonholonomic constraints. Non-smooth constraints associated with some kinematic inequalities are treated by Godoy and Mirasso [13]. The Lagrange multiplier $\lambda$ can be introduced for the constrained system as:

$\underline{M} \underline{\ddot{x}}+\underline{K} \underline{x}+\lambda \underline{\alpha}=\underline{0}$.

The new dynamical constrained system can be characterized by a system of dimension $n+1$ :

$\underline{\underline{\tilde{M}}} \underline{\ddot{y}}+\underline{\tilde{K}}(\underline{\alpha}) \underline{y}=\underline{0} \quad$ with $\underline{\underline{\tilde{M}}}(\underline{\alpha})=\left(\begin{array}{ll}\underline{\underline{M}} & \underline{0} \\ \underline{0}^{T} & 0\end{array}\right)$,

$\underline{\underline{K}}(\underline{\alpha})=\left(\begin{array}{cc}\underline{\underline{K}} & \underline{\alpha} \\ \underline{\alpha}^{T} & 0\end{array}\right)$ and $\quad \underline{y}=\left(\frac{x}{\lambda}\right)$.

Assuming that no flutter instabilities prevail in this constrained system, the loss of stability is given by the singularity condition dealing with the matrix of dimension $n+1$ :

$\operatorname{det}(\underline{\tilde{K}}(\underline{\alpha}))=0$.

Therefore, the stability criterion of the constrained system is naturally affected by the choice of the given structural parameter $\alpha$.
According to the implicit function theorem, the stability criterion given by Eq. (8) locally gives the buckling load as a function of the kinematics parameters parameterized by the vector $\underline{\alpha}$ :

$\left|\begin{array}{cc}\underline{\underline{K}}(p) & \underline{\alpha} \\ \underline{\alpha}^{T} & 0\end{array}\right| \Rightarrow p=p(\underline{\alpha})$

The stability problems considered in the paper are based on a linearly dependence of the stiffness matrix on the buckling load (see for instance [14]).

\section{The optimization problem}

We are interested by the optimization problem of investigating the optimal buckling load with respect to the kinematics parameters parameterized by the vector $\alpha$. Note that the optimization of Ziegler's column with respect to the minimal volume of the structure has been studied by Gajewski and Zyczkowski [4]. We also mention the works of Pantelides [15] on the minimization of buckling load of conservative discrete systems with uncertainty in the spring stiffnesses. Liu et al. [16] optimizes the intermediate support to maximize the buckling load of continuous columns with conservative loading.

In the problem investigated in the paper, we are searching the optimal parameter $\underline{\alpha}$ with respect to the buckling load (minimization or maximization problem). The applications of such a question can be found for instance in soil mechanics, where the loading test is composed of active loading with kinematics constraints and the critical kinematics parameters are searched for. The kinematics constraints can be associated to the concept of mixed perturbation (see for instance [17-19] or [20]) where some kinematics parameters are imposed during the loading process. In soil mechanics, the failure phenomenon is intimately related to the loading (or control) parameters, applied to the boundary of the geomaterial specimen considered [18]. To exemplify, the case of the isochoric biaxial test can be commented. In this test, a deviatoric stress rate is imposed whereas the "volume" is assigned to remain constant. One control parameter is expressed with stress components, the second one with strain components. Such a loading process can be viewed as a mixed control program, with additional kinematics constraints. Therefore, the study of the effect of additional constraints on the stability boundary of general structures may find applications also in the field of soil mechanics.

This mechanical problem is finally related to what can be called an optimization or an antioptimization problem (see for instance [6]).

Therefore, we are looking for the critical parameter $\underline{\alpha}^{*}$ such as:

$\underline{\alpha}^{*} / \frac{\partial p}{\partial \underline{\alpha}}=\underline{0}$.

We show in this paper that this critical constraint is associated with the so-called second-order work, or the vanishing of the determinant of the symmetric part of the matrix $K$ (see Eq. (3)).

The reasoning is based on the decomposition formulae:

$$
\left(\begin{array}{cc}
\underline{\underline{K}} & \underline{\alpha} \\
\underline{\alpha}^{T} & 0
\end{array}\right) \cdot\left(\begin{array}{cc}
\underline{\underline{K}}^{-1} & -\underline{\underline{K}}^{-1} \underline{\alpha} \\
\underline{0}^{T} & 1
\end{array}\right)=\left(\begin{array}{cc}
\underline{\underline{1}}^{T} & \underline{0} \\
\underline{\alpha}^{T} \underline{\underline{K}}^{-1} & -\underline{\alpha}^{T} \underline{\underline{K}}^{-1} \underline{\alpha}
\end{array}\right)
$$

if $\underline{K}$ is invertible. Using Eq. (11), the determinant of the matrix of the constrained problem $\underline{\underline{K}}(\underline{\alpha})$ leads to simplified result:

$$
\begin{aligned}
\operatorname{det}(\underline{\underline{\tilde{K}}}(\underline{\alpha}))= & -\operatorname{det} \underline{\underline{K}} \operatorname{det}\left(\underline{\alpha}^{T} \underline{\underline{K}}^{-1} \underline{\alpha}\right)=-\underline{\alpha}^{T} \underline{\underline{K}}^{-1} \underline{\alpha} \operatorname{det} \underline{\underline{K}} \\
& \text { if } \operatorname{det} \underline{\underline{K}} \neq 0 .
\end{aligned}
$$


Eq. (12) can be also found directly from Schur complementary formulae. The buckling load $p$ is a function of the parameter $\alpha$, via the implicit function defined by Eq. (9). The minimization of $p$ with respect to $\alpha$ can then be computed directly from the implicit function via:

$\frac{\partial p}{\partial \underline{\alpha}}=\underline{0} \Rightarrow \frac{\partial \operatorname{det}(\underline{\tilde{K}}(\underline{\alpha}))}{\partial \underline{\alpha}}=\underline{0}$.

Such a derivative can be analytically achieved from the expansion of the stability criterion detailed in Eq. (12):

$$
\begin{aligned}
\frac{\partial \operatorname{det}(\underline{\tilde{K}}(\underline{\alpha}))}{\partial \underline{\alpha}} & =-\operatorname{det} \underline{\underline{K}} \frac{\partial\left[\underline{\alpha}^{T} \underline{K}^{-1} \underline{\alpha}\right]}{\partial \underline{\alpha}} \\
& =-2 \operatorname{det} \underline{\underline{K}}\left(\underline{K}^{-1}\right)^{S} \underline{\alpha}=\underline{0} \Rightarrow \operatorname{det} \underline{K}^{S}=0 .
\end{aligned}
$$

Note that det $K \neq 0$ was specifically assumed at the beginning of the reasoning. $\overline{\bar{E} q}$. (14) is based on the remarkable property:

$\operatorname{det}\left(\underline{\underline{K}}^{-1}\right)^{S}=\frac{\operatorname{det} \underline{\underline{\underline{K}}}}{(\operatorname{det} \underline{\underline{K}})^{2}}$.

Therefore, the optimization process of the buckling load with respect to the kinematic constraints leads to the second-order work criterion given by Eq. (3), assuming that only the divergence instabilities are studied. In other words, when considering the minimization problem, the second-order work criterion is associated with the lower bound of the critical load of all the constrained systems. The critical constraint $\alpha^{*}$ (related to a critical instability direction at the material scale when studying material instabilities) has some particular features that we will investigate.

$$
\left(\underline{\underline{K}}^{-1}\right)^{S} \underline{\alpha}^{*}=\underline{0}
$$

This critical kinematics constraint, destabilizing the system, can then be interpreted in term of preferentially instability direction (at the material scale, for instance in soil mechanics). In soil mechanics, assuming a material point problem, the tangent stiffness matrix corresponds to the constitutive relation. The critical kinematics constraint appearing in Eq. (16) corresponds to the instability direction at the material scale for mixed loading parameters (see [18]).

Eq. (12) is based on the invertibility condition of the stiffness matrix of the unconstrained system (det $\underline{\underline{K}} \neq 0$ ). The stability problem of the constrained system in the particular case where the stiffness matrix is not invertible (det $\underline{K}=0$ ), i.e. at the stability boundary of the free system can be investigated from the buckling mode of the free system:

$$
\operatorname{det} \underline{\underline{K}}=0 \Rightarrow \exists \underline{x^{*}} \neq \underline{0} / \underline{K} \cdot \underline{x}^{*}=\underline{0} \text {. }
$$

The static equations of the constrained system expressed by Eq. (6) are also valid for the divergence instability:

$\underline{K} \cdot \underline{x}+\lambda \underline{\alpha}=\underline{0} \quad$ and $\quad \underline{x}^{T} \cdot \underline{\alpha}=0$.

It is clear in this particular case that the free buckling mode is solution of the constrained system with $\lambda=0$. i.e. a null Lagrange multiplier:

$\underline{x}=\underline{x}^{*}, \quad \lambda=0$ and $\underline{x}^{* T} \cdot \underline{\alpha}=0$.

Such a particular case only concerns the intersection of the stability boundary of the free system det $\underline{K}=0$ with the one of the constrained system det $\underline{\underline{\tilde{K}}}(\underline{\alpha})=0$.

\section{Some implications for conservative and non-conservative systems}

\subsection{Conservative systems}

For conservative systems, the stability criterion is given by the vanishing of the determinant of the stiffness matrix $\left(\operatorname{det}(\underline{\underline{K}})=\operatorname{det}\left(\underline{\underline{K}}^{S}\right)=0\right)$. The optimization of the buckling load associated with the kinematically constrained system leads to the same criterion, namely $\left(\operatorname{det}(\underline{\underline{K}})=\operatorname{det}\left(\underline{\underline{K}}^{S}\right)=0\right)$ for a specific constraint $\alpha^{*}$. As show by Eq. (19), this critical constraint $\alpha^{*}$ is compatible with the buckling mode $\underline{x}^{*}$ of the free system in this case:

$\underline{\alpha}^{* T} \cdot \underline{x}^{*}=0$.

Considering the minimization problem, the first buckling mode of the free system has to be considered for the critical constraint. Therefore, the minimization of the constrained system can be obtained from the shape of the fundamental buckling mode of the free system, which is an intuitive idea.

In the presence of conservative systems, these results mean that a constraint necessarily stabilizes the free system (see also [2] for a general discussion of this theorem; see also [21]). Eventually, the critical constraint (associated with the minimization problem) does not affect the stability of the constrained system. This also means that the stability domain of the unconstrained system is included in the one of the constrained systems in the conservative case.

Note that this property even in case of conservative systems is no more guaranteed if the equilibrium position depends on the loading range. We keep in mind that in some cases an increase in stiffness in a structure may also decrease the buckling load, even for conservative systems ([22-25] or [26]). Considering now the maximization problem, the critical constraint is obtained from the highest buckling mode.

\subsection{Non-conservative systems}

For non-conservative elastic systems (without constraints), the static criterion also holds in cases of divergence instabilities $(\operatorname{det}(\underline{\underline{K}})=0)$. For this type of instability, the second-order work criterion $\left(\operatorname{det}\left(\underline{\underline{K}}^{S}\right)=0\right)$ constitutes a lower bound of the stability boundary [9]. In cases of flutter instability, no theorem guarantees the lower bound status of the second-order work criterion. In other words, only flutter instabilities can precede the second-order work criterion.

Effects of additional constraints for non-conservative systems are no more intuitive. We focus here on the minimization problem with respect to the kinematics parameters. As the secondorder work criterion $\left(\operatorname{det}\left(\underline{\underline{K}}^{S}\right)=0\right)$ constitutes a lower bound of the stability boundary $(\operatorname{det}(\underline{K})=0)$, this means that for non-conservative systems, there exists necessarily a kinematics constraint $\alpha$ which destabilizes the constrained system. Therefore, a specific kinematics constraint can stabilize or destabilize a nonconservative system (we will give some specific examples in this paper). However, for all kinematics constraints, there necessarily exists a constraint which destabilizes the non-conservative system. The constraint $\underline{\alpha}^{*}$ associated to the lowest critical load is obtained from the second-order work criterion. Excluding flutter instabilities, the second-order work criterion is not only a lower bound of the stability boundary of the free system, but also the boundary of the stability domain, for all mixed perturbations based on proportional kinematics conditions. 


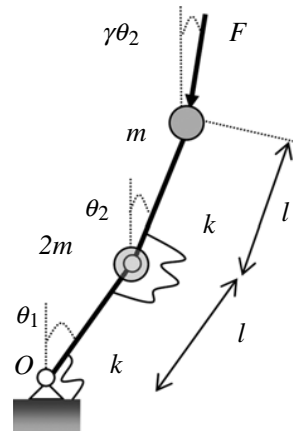

Fig. 1. Ziegler's model under partial follower load-free system.

\section{A structural example-Ziegler's column}

The Ziegler column, loaded by a partial follower load $[27,8]$ can be considered as an interesting structural system, because instability by divergence and instability by flutter may both appear, depending on the structural parameters. This undamped structural system is sometimes called a generalised Ziegler column. It is a pinned column with a sub-tangential or super-tangential buckling load $F$ (sub-tangential for $\gamma<1$, super-tangential for $\gamma>1$ ). This is a two-degrees-of-freedom system with a state vector $x^{T}=$ $\left(\theta_{1}, \theta_{2}\right)$, where $\theta_{i}$ is the rotation in each spring (see Fig. 1$)$. The equilibrium position is given by $\left(\theta_{1}, \theta_{2}\right)=(0,0)$. The stiffness of each spring is denoted by $k$.

The elastic potential $V$ of this system can be written as:

$V=\frac{1}{2} k \theta_{1}^{2}+\frac{1}{2} k\left(\theta_{2}-\theta_{1}\right)^{2}$.

The virtual work of external forces is given by:

$\delta W=F \cos \left(\gamma \theta_{2}\right) \delta u-F \sin \left(\gamma \theta_{2}\right) \delta v$

with

$\left\{u=2 l-l \cos \theta_{1}-l \cos \theta_{2}\right.$

$\left\{v=l \sin \theta_{1}+l \sin \theta_{2}\right.$

This variation can be also presented in the condensed form:

$\delta W=F l \sin \left(\theta_{1}-\gamma \theta_{2}\right) \delta \theta_{1}+F l \sin \left(\theta_{2}-\gamma \theta_{2}\right) \delta \theta_{2}$.

Clearly, this system is a conservative system only when $\gamma=0$. The stiffness matrix $\underline{K}$ is obtained from the linearized equations around the equilibrium position $\left(\theta_{1}, \theta_{2}\right)=(0,0)$ :

$\delta V-\delta W=\underline{x}^{T} \underline{\underline{K}}^{T} \delta \underline{x} \quad$ with $\underline{x}=\left(\begin{array}{l}\theta_{1} \\ \theta_{2}\end{array}\right)$.

The stiffness matrix is then written as (see also [8]):

$\underline{\underline{K}}=\left(\begin{array}{cc}2 k-F l & -k+F l \gamma \\ -k & k-F l(1-\gamma)\end{array}\right) \quad$ or

$\underline{\underline{K}}=k\left(\begin{array}{cc}2-p & \gamma p-1 \\ -1 & 1-(1-\gamma) p\end{array}\right) \quad$ with $p=\frac{F l}{k}$

$p$ is the loading parameter and $\gamma$ is the parameter that characterises the orientation of the follower load (see Fig. 1). $\gamma=0$ corresponds to the conservative case and $\gamma=1$ to the academic case of Ziegler's column. The mass matrix is given for instance by [8]:

$\underline{\underline{M}}=m l^{2}\left(\begin{array}{ll}3 & 1 \\ 1 & 1\end{array}\right)$.

The dynamics stability of this non-conservative system is treated by Hermann and Bungay [27], including flutter instabilities.

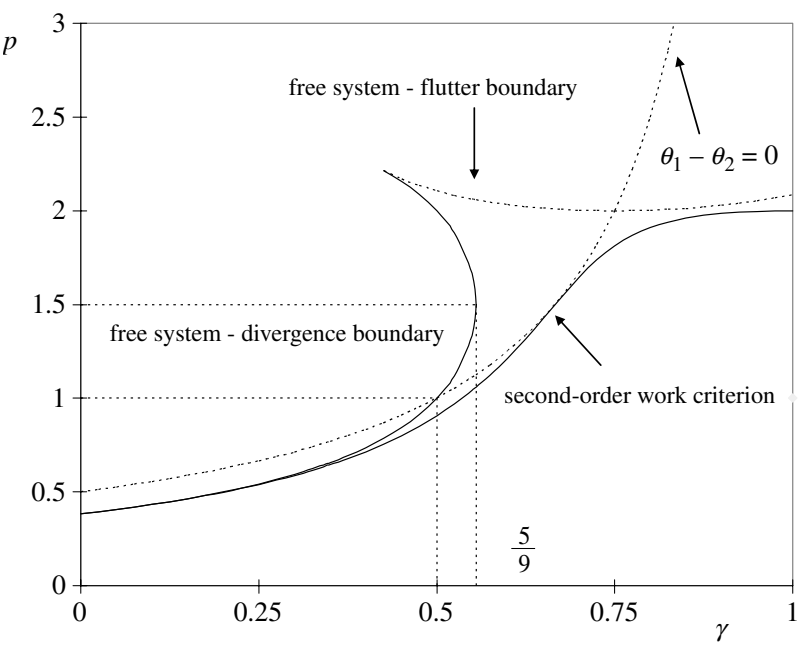

Fig. 2. Comparison of the instability load of the free system and the constraint system.

The flutter load is calculated as [27]:

$p=\frac{8-\gamma \pm \sqrt{(8-\gamma)^{2}-41\left(1+(1-\gamma)^{2}\right)}}{2\left[1+(1-\gamma)^{2}\right]}$.

The "static" criterion can be applied to characterise the instability boundary by divergence of the free system (Eq. (2)):

$\operatorname{det}(\underline{\underline{K}})=0 \Leftrightarrow(1-\gamma) p^{2}-3 p(1-\gamma)+1=0$.

The linearized constraints studied in the paper are written for the structural model considered in this part as:

$\alpha_{1} \theta_{1}+\alpha_{2} \theta_{2}=0$.

The instability boundary of the constrained system necessarily arises by divergence ( single-degree-of-freedom system), leading to the instability load, obtained from Eq. (8):

$\operatorname{det}(\underline{\underline{\tilde{K}}}(\underline{\alpha}))=0 \Leftrightarrow p\left(\alpha_{1}, \alpha_{2}\right)=\frac{\alpha_{1}^{2}+2 \alpha_{1} \alpha_{2}+2 \alpha_{2}^{2}}{\alpha_{1}^{2}(1-\gamma)+\gamma \alpha_{1} \alpha_{2}+\alpha_{2}^{2}}$.

For instance, a particular case is the constrained system that has one spring with infinite stiffness $\left(\theta_{1}-\theta_{2}=0\right)$ :

$\theta_{1}-\theta_{2}=0 \Rightarrow \alpha_{1}=-\alpha_{2}=1 \Rightarrow p=\frac{1}{2(1-\gamma)}$.

In Fig. 2, the divergence boundary of the free system is plotted, and compared to the instability boundary of the constrained system.

It is observed that there is a region $\gamma \in[1 / 2 ; 3 / 4]$ where the system with an infinite stiffness for one spring, leading to $\theta_{1}=\theta_{2}$, has a lower instability load than the initial one, even in the divergence transition area $\gamma \in[1 / 2 ; 5 / 9]$. For this nonconservative system, an increase in stiffness may destabilize the system, even if only divergence instabilities are considered. This is typically a particular feature of the non-conservative nature of the system. Indeed, in case of conservative systems, an increase of stiffness generally leads to an increase of the buckling load (or the natural vibrations-see Rayleigh's theorem [26]). Note that this property even in case of conservative systems is no more guaranteed if the equilibrium position depends on the loading range. We keep in mind that in some cases an increase in stiffness in a structure may also decrease the buckling load, even for conservative systems ([22-25] or [26]). 
In other words, a particular kinematics constraint can stabilize or destabilize a non-conservative system (we give some specific examples in this paper). The second-order work criterion (Eq. (3)) leads to the lower bound of the parameterized instability load:

$\operatorname{det}\left(\underline{\underline{K}}^{S}\right)=0 \Leftrightarrow\left(1-\gamma-\frac{\gamma^{2}}{4}\right) p^{2}-3 p(1-\gamma)+1=0$.

It is easy checked in Fig. 2 that the particular constrained system by Eq. (31) leads to a stability boundary which is tangent to the second-order criterion (Eq. (32)) for a critical parameter $\gamma$. This is the geometrical interpretation that the second-order work criterion corresponds to the intersection of all stability domains parameterized by the kinematics constraint (when stability prevails by divergence).

Application of Eq. (13) to this two-degree-of-freedom system leads to the coupled system:

$\frac{\partial}{\partial \alpha_{1}}\left|\begin{array}{ccc}K_{11} & K_{12} & \alpha_{1} \\ K_{21} & K_{22} & \alpha_{2} \\ \alpha_{1} & \alpha_{2} & 0\end{array}\right|=0$ and $\frac{\partial}{\partial \alpha_{2}}\left|\begin{array}{ccc}K_{11} & K_{12} & \alpha_{1} \\ K_{21} & K_{22} & \alpha_{2} \\ \alpha_{1} & \alpha_{2} & 0\end{array}\right|=0$.

It is easy to develop these two systems in a linear system of two equations with two unknowns $\left(\alpha_{1}, \alpha_{2}\right)$ :

$\int-\left(K_{12}+K_{21}\right) \alpha_{2}+2 K_{22} \alpha_{1}=0$

$\left\{2 K_{11} \alpha_{2}-\left(K_{12}+K_{21}\right) \alpha_{1}=0\right.$.

Obviously, Eq. (3) is found again from Eq. (34), and more specifically the second-order work criterion for a two-dimensional system:

$K_{11} K_{22}-\left(\frac{K_{12}+K_{21}}{2}\right)^{2}=0 \Leftrightarrow \operatorname{det}\left(\underline{\underline{K}}^{S}\right)=0$.

For the present problem, the critical parameter $\alpha^{*}$ is then computed from Eq. (34) as:

$\frac{\alpha_{2}}{\alpha_{1}}=\frac{\gamma p-2}{2(2-p)}$ with $p\left(\alpha_{1}, \alpha_{2}\right)=\frac{\alpha_{1}^{2}+2 \alpha_{1} \alpha_{2}+2 \alpha_{2}^{2}}{\alpha_{1}^{2}(1-\gamma)+\gamma \alpha_{1} \alpha_{2}+\alpha_{2}^{2}}$.

Eq. (36) can be expressed in a single equation dealing with the kinematics constraints:

$2 \alpha_{2}\left[2-p\left(\alpha_{1}, \alpha_{2}\right)\right]=\alpha_{1}\left[\gamma p\left(\alpha_{1}, \alpha_{2}\right)-2\right]$.

This is equivalent to the second-order polynomial equation for the critical set of parameters (the third-order terms are vanishing):

$2(\gamma-1)\left(\frac{\alpha_{2}}{\alpha_{1}}\right)^{2}+2(1-2 \gamma) \frac{\alpha_{2}}{\alpha_{1}}+(2-3 \gamma)=0$

whose solution is given for the minimization problem by:

$\left(\frac{\alpha_{2}}{\alpha_{1}}\right)^{*}=\frac{2 \gamma-1+\sqrt{(2 \gamma-1)^{2}-(2 \gamma-2)(2-3 \gamma)}}{2(\gamma-1)} \leq 0$.

The other solution leads to the maximization problem:

$\left(\frac{\alpha_{2}}{\alpha_{1}}\right)^{*}=\frac{2 \gamma-1-\sqrt{(2 \gamma-1)^{2}-(2 \gamma-2)(2-3 \gamma)}}{2(\gamma-1)} \geq 0$.

The critical parameter $\underline{\alpha}^{*}$ depends on the non-conservative structural parameter $\gamma$ for this problem. Introduction of this critical parameter in Eq. (30) necessarily leads to the second-order work criterion (Eq. (32)).

This result shows the strong link between the concept of critical kinematics constraint and the direction of loading in the space of incremental strain, leading to the vanishing of the second-order criterion.

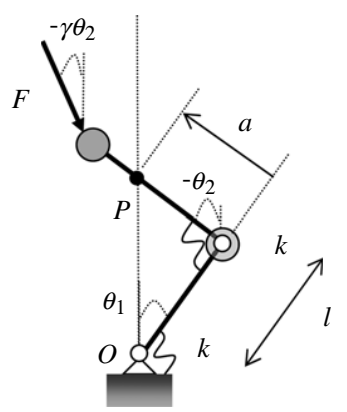

Fig. 3. Ziegler's model under partial follower load-free system with kinematics constraint; case $a>0$.

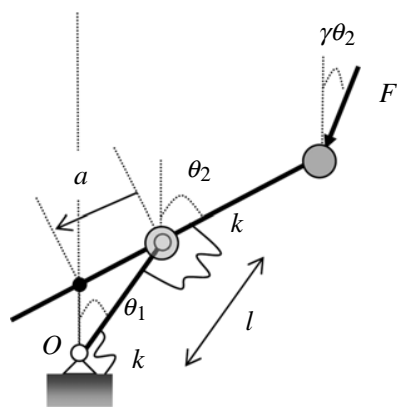

Fig. 4. Ziegler's model under partial follower load-free system with kinematics constraint; case $a<0$.

\section{Physical meaning of additional constraints}

We show now how a kinematic constraint can be introduced in the studied problem. Let us consider that the point $P$ (parameterized by the distance $a$ from the second spring) to move along a vertical axis (see for instance [28]). Figs. 3 and 4 show two basic examples of such a constraint. The constraint on the lateral displacement of point $P$ can be expressed by:

$l \sin \theta_{1}+a \sin \theta_{2}=0$

leading to the constrained linearized kinematics equation:

$\alpha_{1} \theta_{1}+\alpha_{2} \theta_{2}=0$ with $\frac{\alpha_{2}}{\alpha_{1}}=\frac{a}{l}$.

Fig. 3 shows an example with a positive value for $a(a>0)$, whereas Fig. 4 shows an example with a negative value for $a(a<0)$. Typically, for sufficiently small values of the nonconservative parameter $\gamma$, Fig. 3 typically corresponds to the maximization problem whereas Fig. 4 corresponds to the minimization problem. This is for instance the case of the conservative problem $(\gamma=0)$ :

$\left(\frac{\alpha_{2}}{\alpha_{1}}\right)_{\min }^{*}=\frac{1-\sqrt{5}}{2} \approx-0.618$

and

$\left(\frac{\alpha_{2}}{\alpha_{1}}\right)_{\max }^{*}=\frac{1+\sqrt{5}}{2} \approx 1.618$.

Note of course that these values are the exact ones of the buckling modes of the free system (without additional constraints). This optimization process is clearly shown in Fig. 5 for the conservative case. The second-order work criterion corresponds to the minimization and the maximization problem. Fig. 5 shows that the stability boundary of the constrained system is above the one of the free system, and is tangent to the boundary of the free 


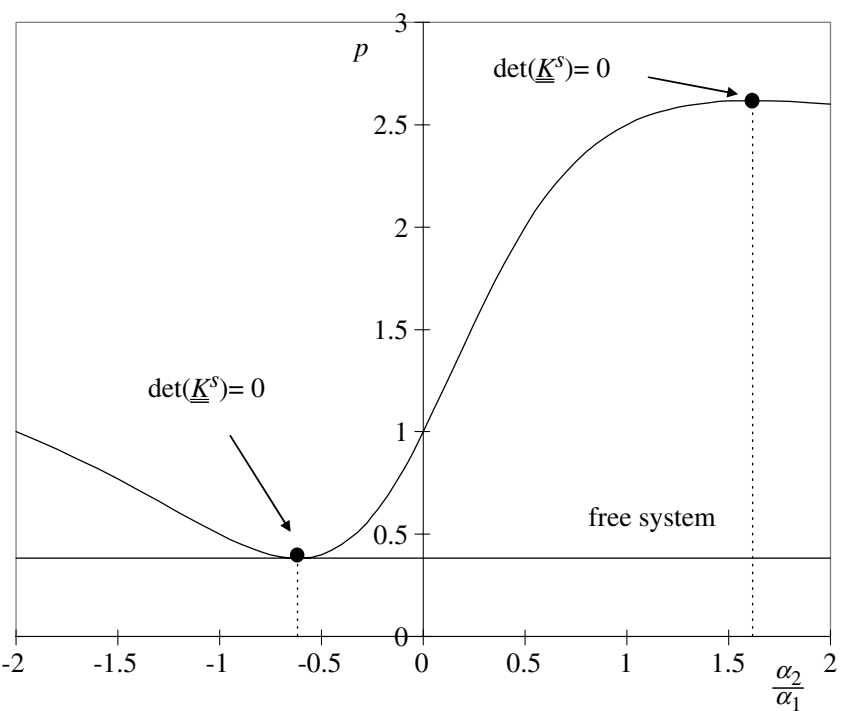

Fig. 5. Effect of an additional constraint on the buckling load; conservative system $\gamma=0$.

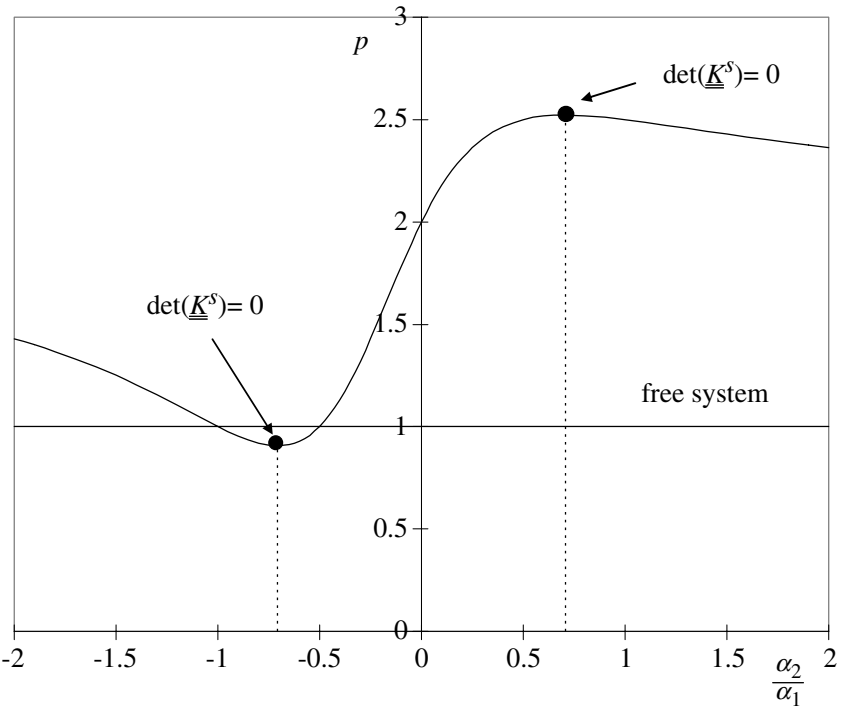

Fig. 6. Effect of an additional constraint on the buckling load; non-conservative system $\gamma=0.5$.

system at the critical point corresponding to the minimization problem (see Eq. (43) for the conservative system). It has to be outlined that an additional constraint cannot destabilize the system for the conservative problem (see also Fig. 5). This property is not true for the non-conservative problem, where an additional constraint can destabilize the structural system (see Fig. 6). There is clearly a destabilizing zone around the critical point (associated to the second-order work criterion) where the boundary of the constrained system is below the one of the free system. Finally, the location of the optimum values of the kinematics constraints are shown in Fig. 7.

\section{Conclusions}

For conservative systems, the stability criterion is given by the vanishing of the determinant of the stiffness matrix $\left(\operatorname{det}(\underline{\underline{K}})=\operatorname{det}\left(\underline{\underline{K}}^{S}\right)=0\right)$. For non-conservative elastic systems,

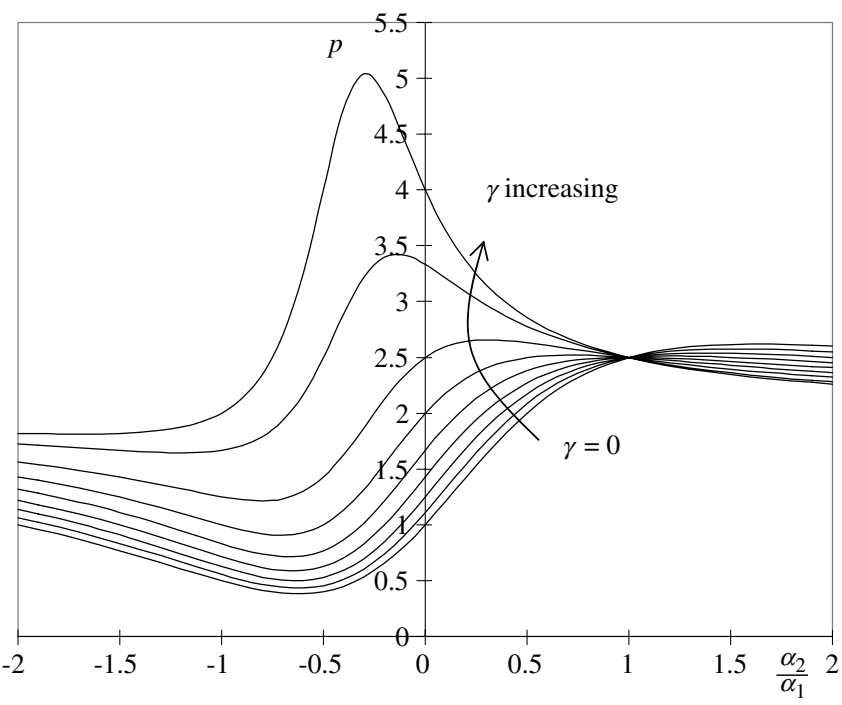

Fig. 7. Effect of an additional constraint on the buckling load; parametric study; $\gamma \in\{0 ; 0.1 ; 0.2 ; 0.3 ; 0.4 ; 0.5 ; 0.6 ; 0.7 ; 0.75\}$.

the static criterion also holds in cases of divergence instabilities $(\operatorname{det}(\underline{\underline{K}})=0)$. For this type of instability, the second-order work criterion $\left(\operatorname{det}\left(\underline{\underline{K}}^{S}\right)=0\right)$ constitutes a lower bound of the stability boundary [9]. In cases of flutter instability, no theorem guarantees the lower bound status of the second-order work criterion. In other words, only flutter instabilities can precede the second-order work criterion.

We question in this paper the meaning of the second-order work criterion for the dynamics system under kinematics constraints. It is shown that the second-order work criterion corresponds to the intersection of all stability domains parameterized by the kinematics constraint (when stability prevails by divergence). More specifically, the vanishing of the second-order work criterion corresponds to the critical kinematics constraint, which can be interpreted as an instability direction when the material stability analysis is considered. The second-order work criterion $\left(\operatorname{det}\left(\underline{K}^{S}\right)=0\right)$ is related to the minimization of the buckling load with respect to the kinematics constraints, but also to the maximization of the buckling load.

As a consequence, a particular kinematics constraint can stabilize or destabilize a non-conservative system (we give some specific examples in this paper). However, for all kinematics constraints, there necessarily exists a constraint which destabilizes the non-conservative system. The constraint associated to the lowest critical load is associated with the second-order criterion. In the case of a conservative system, this means that a constraint necessarily stabilizes the free system (see also [2]). Note that this property even in case of conservative systems is no more guaranteed if the equilibrium position depends on the loading range. We keep in mind that in some cases an increase in stiffness in a structure may also decrease the buckling load, even for conservative systems ([22-25] or [26]). This phenomenon was also observed in non-conservative systems controlled by the flutter phenomenon [29].

Excluding flutter instabilities, the second-order work criterion is not only a lower bound of the stability boundary of the free system, but also the boundary of the stability domain, for all mixed perturbations based on proportional kinematics conditions. The conclusions of this paper, and in particular the stability boundary of such non-conservative systems maybe strongly affected by 
infinitesimal damping (see for instance [1,5] or [30]). Furthermore, infinitesimal damping may also exhibit periodic attractors (flutter instability) for a load smaller than that of static instability (divergence instability) [31].

\section{Acknowledgement}

The authors wish to thank Prof. Vigen Arakelian for helpful discussions about the structural design including kinematics constraints.

\section{References}

[1] Bolotin VV. Nonconservative problems of the theory of elastic stability. New York: Pergamon Press; 1963.

[2] Ziegler H. Principles of structural stability. Blaisdell Publishing Company; 1968.

[3] Challamel N, Casandjian C, Lerbet J. On the occurence of flutter in the lateral-torsional instabilities of circular arches under follower loads. J Sound Vib 2009;320:617-31.

[4] Gajewski A, Zyczkowski M. Optimal structural design under stability constraints. Dordrecht (The Netherlands): Kluwer Academic Publishers; 1988.

[5] Seyranian AP, Mailybaev AA. Multiparameter stability theory with mechanical applications. World Scientific; 2003.

[6] Elishakoff I, Ohsaki M. Optimization and anti-optimization of structures under uncertainty. World Scientific; 2010.

[7] La Salle J, Lefschetz S. Stability by Liapunov's direct method with applications. New York: Academic Press; 1961.

[8] Leipholz H. Stability theory. New York: John Wiley; 1987.

[9] Huseyin K, Leipholz H. Divergence instability of multiple-parameter circulatory systems. Quart Appl Math 1973;185-97.

[10] Bažant ZP, Cedolin L. Stability of structures-elastic, inelastic, fracture, and damage theories. New York: Dover Publications, Inc.; 2003.

[11] Challamel N. Comments on the paper "Instability of elastic bodies" written by Elie Absi and Jean Lerbet. Mech Res Comm 2004;31(1):39-44; Mech Res Comm 2005;32:610-2.

[12] Roithmayr CM, Hodges DH. An argument against augmenting the Lagrangean for nonholonomic systems. J Appl Mech 2009;76: 034501:1-3.
[13] Godoy LA, Mirasso AE. On the elastic stability of static non-holonomic systems. Internat J Solids Structures 2003;40:3439-62.

[14] Tarnai T. The southwell and the Dunkerley theorems. In: Tarnai T, editor. Summation theorems in structural stability. CISM course, vol. 354. Wien: Springer-Verlag; 1995. p. 141-85.

[15] Pantelides CP. Buckling of elastic columns using convex model of uncertain springs. J Eng Mech 1995;121(7):837-44.

[16] Liu ZS, Hu HC, Huang C. Derivative of buckling load with respect to support locations. J Eng Mech 2000;126(6):559-64.

[17] Absi E, Lerbet J. Instability of elastic bodies. Mech Res Comm 2004;31(1): 39-44.

[18] Nicot F, Darve F, Khoa HDV. Bifurcation and second-order work in geomaterial. Int J Numer Anal Methods Geomech 2007;31(8):1007-32.

[19] Lerbet J, Absi E, Rigolot A. About the stability of nonconservative undamped elastic systems: some new elements. Int J Struct Stab Dyn 2009;9(2):357-67.

[20] Challamel N, Nicot F, Lerbet J, Darve F. On the stability of non-conservative systems under mixed perturbations. Eur J Environ Civil Eng 2009;13(3): 347-67.

[21] Weinberger H. Some mathematical aspects of buckling. In: Tarnai T, editor. Summation theorems in structural stability. CISM courses and lectures, vol. 354. Wien, (NY): Springer-Verlag; 1995. p. 1-37.

22] Parnes R. A paradoxical case in a stability analysis. AIAA J 1977;15:1533-4

[23] Tarnai T. Destabilizing effect of additional restraint on elastic bar structures. Int J Mech Sci 1980;22:379-90.

[24] Panovko YG, Sorokin SV. Paradoxical influence of an increase of rigidity on buckling loads and natural frequencies for elastic systems. Mech Res Comm 1993;20(1):9-14

[25] Milner HR. Stability of discrete systems. In: Tarnai T, editor. Summation theorems in structural stability. CISM course, vol. 354. Wien: Springer-Verlag; 1995. p. 39-109.

[26] Tarnai T. Paradoxical behaviour of vibrating systems challenging Rayleigh's theorem. In: 21st international congress of theoretical and applied mechanics. 2004.

[27] Hermann G, Bungay RW. On the stability of elastic systems subjected to nonconservative forces. J Appl Mech 1964;86:435-40.

[28] Arakelian V. Structure et cinématique des mécanismes. Hermes; 1997.

[29] Sundararajan C. Influence of end supports on the stability of nonconservative elastic systems. J Appl Mech 1974;41:313-5.

[30] Krechetnikov R, Marsden JE. On destabilizing effects of two fundamental nonconservative forces. Physica D 2006;214:25-32.

[31] Kounadis AN. Flutter instability and other singularity phenomena in symmetric systems via combination of mass distribution and weak damping. Internat J Non-Linear Mech 2007;42:24-35. 